\title{
HerMES: Far infrared properties of known AGN in the HerMES fields *
}

\author{
E. Hatziminaoglou ${ }^{1}$, A. Omont ${ }^{2}$, J. A. Stevens ${ }^{3}$, A. Amblard ${ }^{4}$, V. Arumugam ${ }^{5}$, R. Auld ${ }^{6}$, H. Aussel ${ }^{7}$, T. Babbedge ${ }^{8}$,
} A. Blain ${ }^{9}$, J. Bock ${ }^{9,10}$, A. Boselli ${ }^{11}$, V. Buat ${ }^{11}$, D. Burgarella ${ }^{11}$, N. Castro-Rodríguez ${ }^{12}$, A. Cava ${ }^{12}$, P. Chanial ${ }^{8}$, D. L. Clements ${ }^{8}$, A. Conley ${ }^{13}$, L. Conversi ${ }^{14}$, A. Cooray ${ }^{4,9}$, C. D. Dowell ${ }^{9,10}$, E. Dwek ${ }^{15}$, S. Dye ${ }^{6}$, S. Eales ${ }^{6}$, D. Elbaz ${ }^{7}$, D. Farrah ${ }^{16}$, M. Fox ${ }^{8}$, A. Franceschini ${ }^{17}$, W. Gear ${ }^{6}$, J. Glenn ${ }^{13}$, E. A. González Solares ${ }^{18}$, M. Griffin ${ }^{6}$, M. Halpern ${ }^{19}$, E. Ibar $^{20}$, K. Isaak ${ }^{6}$, R. J. Ivison ${ }^{20,5}$, G. Lagache ${ }^{21}$, L. Levenson ${ }^{9,10}$, N. Lu ${ }^{9,22}$, S. Madden ${ }^{7}$, B. Maffei ${ }^{23}$, G. Mainetti ${ }^{17}$, L. Marchetti ${ }^{17}$, A. M. J. Mortier ${ }^{8}$, H. T. Nguyen ${ }^{9,10}$, B. O'Halloran ${ }^{8}$, S. J. Oliver ${ }^{16}$, M. J. Page ${ }^{24}$, P. Panuzzo , A. Papageorgiou ${ }^{6}$, C. P. Pearson ${ }^{25,26}$, I. Pérez-Fournon ${ }^{12}$, M. Pohlen 6 , J. I. Rawlings ${ }^{24}$, D. Rigopoulou ${ }^{25,27}$, D. Rizzo ${ }^{8}$, I. G. Roseboom ${ }^{16}$, M. Rowan-Robinson ${ }^{8}$, M. Sanchez Portal ${ }^{14}$, B. Schulz ${ }^{9,22}$, D. Scott ${ }^{19}$, N. Seymour ${ }^{24}$, D. L. Shupe ${ }^{9,22}$, A. J. Smith ${ }^{16}$, M. Symeonidis ${ }^{24}$, M. Trichas ${ }^{8}$, K. E. Tugwell ${ }^{24}$, M. Vaccari ${ }^{17}$, I. Valtchanov ${ }^{14}$,

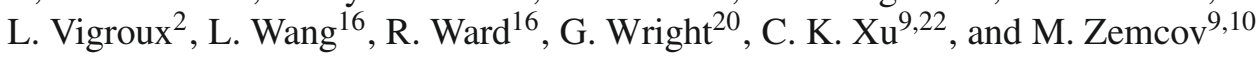

(Affiliations are available in the online edition)

Received 31 March 2010 / Accepted 19 April 2010

\section{ABSTRACT}

Nuclear and starburst activity are known to often occur concomitantly. Herschel-SPIRE provides sampling of the far-infrared (FIR) spectral energy distributions (SEDs) of type 1 and type 2 AGN, allowing for the separation between the hot dust (torus) and cold dust (starburst) emission. We study large samples of spectroscopically confirmed type 1 and type 2 AGN lying within the Herschel Multi-tiered Extragalactic Survey (HerMES) fields observed during the science demonstration phase, aiming to understand their FIR colour distributions and constrain their starburst contributions. We find that one third of the spectroscopically confirmed AGN in the HerMES fields have $5 \sigma$ detections at $250 \mu$ m, in agreement with previous (sub)mm AGN studies. Their combined Spitzer-MIPS and Herschel-SPIRE colours (specifically $S_{250} / S_{70}$ vs $S_{70} / S_{24}$ ) quite clearly separate them from the non-AGN, star forming galaxy population, as their $24 \mu \mathrm{m}$ flux is dominated by the hot torus emission. However, their SPIRE colours alone do not differ from those of non-AGN galaxies. SED fitting shows that all those AGN need a starburst component to fully account for their FIR emission. For objects at $z>2$ we find a correlation between the infrared luminosity attributed to the starburst component, $L_{\mathrm{SB}}$, and the AGN accretion luminosity, $L_{\mathrm{acc}}$, with $L_{\mathrm{SB}} \propto L_{\mathrm{acc}}^{0.35}$. Type 2 AGN detected at $250 \mu \mathrm{m}$ show on average higher $L_{\mathrm{SB}}$ than type 1 objects but their number is still too low to establish whether this trend indicates stronger star formation activity.

Key words. galaxies: active - galaxies: Seyfert - galaxies: star formation - infrared: general - quasars: general

\section{Introduction}

Active galactic nuclei (AGN) and starburst activity, both among the most energetic extragalactic phenomena, have been studied separately for decades. However, it is only in the past decade and a half, with the advent of the infrared (IR) observatories (ISO, Spitzer and now Herschel; Pilbratt et al. 2010) that it has become clear that the two phenomena are related and, more often than not, happen concomitantly (e.g. Schweitzer et al. 2006).

The Spitzer IRAC and MIPS cameras sampled the spectral energy distribution (SED) of extragalactic sources exactly where the peak of the AGN dust emission is expected to occur under the paradigm of an axisymmetric dust distribution (often referred to as torus) surrounding the central super-massive black hole. The IRS spectrograph provided the necessary details of the silicate feature in emission and in absorption and we now have a better understanding of the physics of hot dust around AGN

* Herschel is an ESA space observatory with science instruments provided by European-led Principal Investigator consortia and with important participation from NASA. (e.g. Hao et al. 2007; Levenson et al. 2007). The peak of the cold dust emission, however, a tracer of star formation, was beyond the wavelength range explored by Spitzer for high redshift sources and it is only now, with the advent of SPIRE (Griffin et al. 2010), that the full FIR SEDs of galaxies can be built, all the way to $500 \mu \mathrm{m}$.

The work presented here intends to build on the previous experience gathered with Spitzer and BLAST. We study large samples of spectroscopically confirmed type 1 and type 2 AGN in the largest Herschel Multi-tiered Extragalactic Survey (HerMES ${ }^{1}$; Oliver et al., in prep.) fields observed during the science demonstration phase (SDP). The SEDs and IR properties of many of the objects in the samples have been studied in the past (e.g. Richards et al. 2006; Hatziminaoglou et al. 2008, 2009) and their AGN properties are well constrained. The idea is to extend such study to the larger wavelengths now observed by SPIRE in an effort to also constrain the starburst component of these objects.

\footnotetext{
${ }^{1}$ http://hermes.sussex.ac.uk
} 
Table 1. AGN sample reference, field, type, number of objects, objects with $5 \sigma$ detections at $250 \mu \mathrm{m}$ and at both 250 and $350 \mu \mathrm{m}$.

\begin{tabular}{lrrc}
\hline \hline Sample & Type & Nobj & $\begin{array}{c}5 \sigma 250 \mu \mathrm{m} \\
\text { (and } 350 \mu \mathrm{m})\end{array}$ \\
\hline SDSS (LS) & 1 & 168 & $44(26)$ \\
SDSS (FLS) & 1 & 86 & $29(21)$ \\
Papovich et al. (2006) (FLS) & 1 & 159 & $71(42)$ \\
Lacy et al. (2007) (FLS) & 2 & 20 & $5(2)$ \\
Martínez-Sansigre et al. (2006) (FLS) & 2 & 16 & $5(4)$ \\
Polletta et al. (2006) (LS) & 2 & 11 & $2(1)$ \\
Mainieri et al. (2002) (LS) & 2 & 9 & $0(0)$ \\
\hline Total & & 469 & $156(96)$ \\
\hline
\end{tabular}

\section{AGN samples, their SPIRE detections and mid-to-FIR colours}

The AGN master sample considered here consists of a total of 469 spectroscopically confirmed type 1 and type 2 AGN in the Lockman-SWIRE (LS) and the Spitzer First Look Survey (FLS) fields, with redshifts that extend to $z>4$. More specifically, it includes SDSS quasars in LS and FLS; mid-infrared (MIR) selected AGN in FLS with spectroscopic redshifts from the MMT-Hectospec (Papovich et al. 2006); two MIR-selected type 2 AGN samples from Martínez-Sansigre et al. (2006) and Lacy et al. (2007); and two X-ray selected type 2 samples from Mainieri et al. (2002) and Polletta et al. (2006). Their SPIRE fluxes are estimated via linear inversion methods, using, whenever available, the positions of known $24 \mu \mathrm{m}$ sources as priors (see Roseboom et al., in prep.). The details of the various subsamples and the numbers of $250 \mu \mathrm{m}$ and additional $350 \mu \mathrm{m}$ $5 \sigma$ detections are given in Table 1 . Note that none of the X-ray selected type 2 AGN from Mainieri et al. (2002) were detected by SPIRE. All $5 \sigma$ detections at $250 \mu \mathrm{m}$ and $350 \mu \mathrm{m}$ have fluxes above $12.8 \mathrm{mJy}$ and $12.2 \mathrm{mJy}$, respectively. No flux cut is applied at $500 \mu \mathrm{m}$, these fluxes are used, instead, at face value. The different detection rates of the various samples in the two fields are the result of the different depths (FLS being deeper than LS, Oliver et al. 2010, this volume) as well as the selections of the various samples.

It is worth noting that one third of the objects have $5 \sigma$ detections at $250 \mu \mathrm{m}$, also reported by Elbaz et al. (2010). This detection rate is in excellent agreement with results obtained from the studies of bright $\left(M_{B}<-26.1\right)$, high redshift $(z \geq 1.8)$ quasars at $1.2 \mathrm{~mm}$, with the MAx-Planck Millimeter BOlometer (MAMBO) array at IRAM 30-m telescope (Carilli et al. 2001; Omont et al. 2001). It is, however, higher than the $\sim 15 \%$, reported by Priddey et al. (2003) for $1.5<z<3.0$ radio-quiet, luminous $\left(M_{B}<-27.5\right)$ quasars, observed at $850 \mu \mathrm{m}$ with SCUBA on the James Clerk Maxwell Telescope (JCMT). Even though our derived detection rate holds for the whole absolute magnitude range of our sample $\left(-19>M_{g}>-28.3\right)$ as well as in broad $M_{g}$ bins (here we use $M g$, as the SDSS $g$-filter is the closest to the $B$-band used in the other studies), if we look at the more rare, brightest objects $\left(M_{g}<-27.5\right)$ we only find about $20 \%$ of them having $5 \sigma$ detections at $250 \mu \mathrm{m}$, close to the $15 \%$ reported by Priddey et al. (2003).

Though it is difficult to say anything about the significance of the detections of the rest of the objects in an objective way, the observed properties of the AGN with and without SPIRE counterparts are quite similar, both in terms of optical and IR fluxes and in redshift. Therefore, since the present study only makes use of objects with $5 \sigma$ detections at $250 \mu \mathrm{m}$, and since $250 \mu \mathrm{m}$ emission arises from cold dust in the star forming
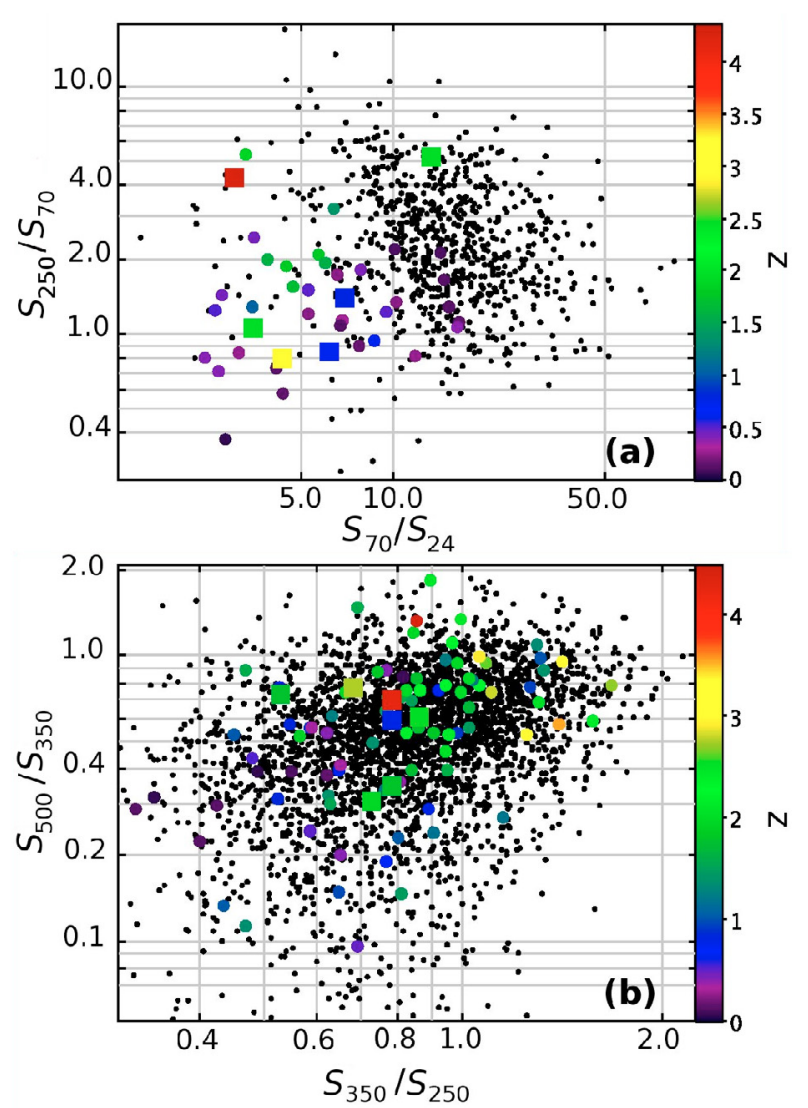

Fig. 1. MIPS-SPIRE colours a) and SPIRE colours b) for type 1 (circles) and type 2 (large squares) AGN as a function of redshift, overplotted on the entire SPIRE catalogue in the FLS field, for objects with $5 \sigma$ detections at $250 \mu \mathrm{m}$ a) and $350 \mu \mathrm{m} \mathrm{b}$ ).

regions, the $250 \mu \mathrm{m}$-detected sample may be biased towards objects with stronger star formation activity. The issue of SPIRE detection rates of AGN is addressed in more detail in Stevens et al., in prep.

Figure 1 shows the MIPS-SPIRE colours $S_{250} / S_{70}$ vs. $S_{70} / S_{24}$ (a) and SPIRE colours $S_{500} / S_{350}$ vs. $S_{350} / S_{250}$ (b) of all AGN with $250 \mu \mathrm{m}$ and $350 \mu \mathrm{m}$ detections above $5 \sigma$ (type 1: circles; type 2: squares) colour-coded based on their redshift, and compared to all SPIRE sources in the FLS field at the same SPIRE detection limits (black dots). AGN have much bluer colours and tend to separate nicely from the bulk of star forming non-active galaxies, as their $24 \mu \mathrm{m}$ fluxes are dominated by the hot dust (torus) emission. This is in agreement with recent MIR spectroscopic studies with Spitzer/IRS showing that the MIR quasar emission mostly arises from the torus, independent on their FIR properties (Netzer et al. 2007). The presence of strong PAH features, however, may slightly weaken this effect in certain redshift ranges. The $7.7 \mu \mathrm{m}$ PAH feature, in particular, might affect the $24 \mu \mathrm{m}$ flux of $z \sim 2$ AGN, when present (Lutz et al. 2008).

In the SPIRE bands the colours of AGN are indistinguishable from those of the star forming non-active galaxies, suggesting that AGN actually appear like starburst galaxies in the FIR. This is consistent with longer wavelength studies that show the FIR emission of submm-luminous quasars and their starburst to share many properties of submm galaxies. Their FIR luminosities are similar, and the best evidence is probably that they follow practically the same FIR/CO luminosity relation as submm galaxies and local ULIRGs (see e.g. Fig. 5 of Riechers et al. 2006). 
A variety of multi-wavelength datasets covering the energy range from the radio to the X-rays is available for the objects in our master sample, but in order to have a uniform wavelength SED coverage to perform the fitting, we only used the available photometry from SDSS DR7 (Abazajian et al. 2009), 2MASS and 2MASSx6 in the Lockman field (Beichman et al. 2003), Spitzer IRAC and MIPS data from the SWIRE (Lonsdale et al. 2004) and FLS Spitzer surveys, and new SPIRE HerMES data. PACS (Poglitsch et al. 2010) data have not been used, as they are only available for parts of the HerMES SDP fields.

\section{Star formation in AGN}

The observed SEDs, described in Sect. 2, were compared, by means of SED fitting with a standard $\chi^{2}$ minimisation, to a series of models comprising three different components: a stellar component composed by various simple stellar population (SSP) models build using the Padova evolutionary tracks (Bertelli et al. 1994); a grid of AGN/torus models that include both a toroidal and a flared disk dust geometry presented in Fritz et al. (2006); and six empirical starburst SEDs. For a full description of the SED fitting and individual model components see Hatziminaoglou et al. (2008, 2009). For reasons explained in detail in Hatziminaoglou et al. (2008) the reduced $\chi^{2}$ can reach high values without undermining our confidence in the fits. We will restrict the present study to the objects with fits having reduced $\chi^{2}<10$.

This leaves a total of 68 (42) type 1 and 11 (7) type 2 AGN, with $5 \sigma$ detections at $250 \mu \mathrm{m}$ (and $350 \mu \mathrm{m}$ ), respectively. As already mentioned, the $500 \mu \mathrm{m}$ are taken at face value, even though the detection level of about one third of them falls below $2 \sigma$. The number drops to about $15 \%$ for the objects with a $5 \sigma$ detection at $350 \mu \mathrm{m}$. Despite the low significance of some of the $500 \mu \mathrm{m}$ data points, they follow nicely the observed SEDs as traced by the other FIR points and are unlikely to affect the fit, because of their large photometric errors. SPIRE $500 \mu \mathrm{m}$ nondetections are not treated as upper limits and are excluded from the fits. Examples fits for a type 1 and a type 2 AGN are shown in Fig. 2.

As a first remark we point out that, in order to reproduce the observed SPIRE data points, a starburst template is always needed, even if we allow for very large (kpc-scale) tori. Large tori are, in any case, unphysical in the sense that they extend well into the host galaxy where other physical phenomena such as star formation may occur, and the AGN is no longer the primary source of dust heating. The SED fitting results in the set of estimations of physical parameters, describing the various components. Here we will focus on the accretion luminosity, $L_{\text {acc }}$, the model luminosity of the accretion disk ranging from soft $\mathrm{X}$-rays to the optical wavelengths scaled to the observed data points, and the IR luminosity of the starburst component, $L_{\mathrm{SB}}$, integrated between restframe 8 and $1000 \mu \mathrm{m}$, as a direct measure of star formation.

Figure 3 shows $L_{\mathrm{SB}}$ as a function of $L_{\mathrm{acc}}$ with each point colour-coded according to redshift for $250 \mu \mathrm{m} 5 \sigma$ detections alone (open symbols) and additional $350 \mu \mathrm{m}$ detections (filled symbols). A broad correlation of $L_{\mathrm{SB}}$ can be seen with $L_{\mathrm{acc}}$, both quantities, however, also scale with redshift, as seen from the colour-coding of the points.

If we divide the sample in bins of redshift, the picture becomes less clear. Table 2 shows the Pearson correlation coefficients for the quantities $L_{\mathrm{SB}}$ and $L_{\mathrm{acc}}$ in five redshift bins. (The missing value in the last column reflects the very small

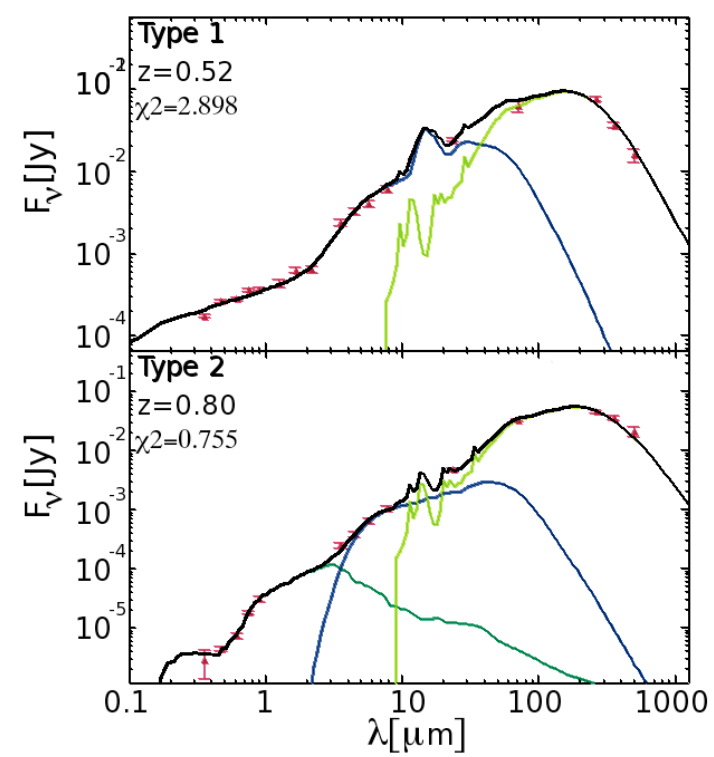

Fig. 2. Example fits for a type 1 (upper panel) and a type 2 (lower panel) AGN. In red the observed data points; in blue the AGN/torus model; in light green the starburst component; in dark green the stellar component, and in black the total model SED. The reported $\chi^{2}$ are reduced.

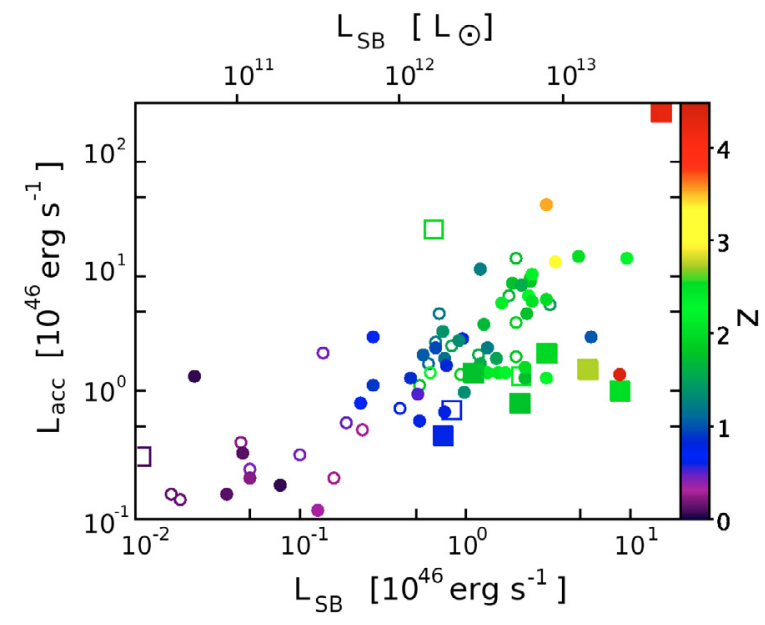

Fig. 3. $L_{\mathrm{SB}}$ as a function of $L_{\mathrm{acc}}$ and redshift (colour-coding) for all the objects (type 1: circles; type 2: large squares) with $5 \sigma$ detections at $250 \mu \mathrm{m}$ and $350 \mu \mathrm{m}$ (filled symbols) and with $5 \sigma$ detections at $250 \mu \mathrm{m}$ alone (additional open symbols).

number of points in the relevant bin, for which a Pearson correlation would be meaningless.) The very low values of the coefficients suggest that the observed global trend may reflect the fact that the sample being flux limited, more distant objects will also be intrinsically more luminous. A lack of correlation between $L_{\mathrm{SB}}$ and $L_{\mathrm{acc}}$ would imply that star formation activity is not influenced by the presence of an active nucleus in the centre of the galaxy. Nevertheless, since the derived $L_{\text {acc }}$ only covers at most two orders of magnitude at any given redshift, and since we are dealing with a limited number of objects, it may be that our sample is not adequate to detect any such correlations. Considering the $z>2.0$ bin alone we do find a $95 \%$ (90\%) probability of $L_{\mathrm{SB}}$ and $L_{\mathrm{acc}}$ correlating as $L_{\mathrm{SB}} \propto L_{\mathrm{acc}}^{0.35}$, for objects with $5 \sigma$ detections at $250 \mu \mathrm{m}$ (and additionally at $350 \mu \mathrm{m}$ ). This value is in surprisingly good agreement with that found by Wang et al. (2008) in their study of $z \sim 6$ quasars with MAMBO.

An important issue in the study of nuclear and star formation activities is that of the occurrence of star formation in the 
Table 2. Correlation coefficients $r$ and $r^{\prime}$ between $L_{\mathrm{SB}}$ and $L_{\text {acc }}$ in bins of objects considering the subsamples with $5 \sigma$ detections at $250 \mu \mathrm{m}$ alone $(N)$ and those with both 250 and $350 \mu \mathrm{m} 5 \sigma$ detections $\left(N^{\prime}\right)$, respectively.

\begin{tabular}{rrrcc}
\hline \hline$z$ & $N$ & $N^{\prime}$ & $r$ & $r^{\prime}$ \\
\hline$z<0.5$ & 15 & 4 & 0.063 & - \\
$0.5<z<1.0$ & 14 & 10 & 0.073 & 0.057 \\
$1.0<z<1.5$ & 9 & 6 & 0.054 & 0.241 \\
$1.5<z<2.0$ & 21 & 13 & 0.109 & 0.241 \\
$z>2.0$ & 18 & 16 & 0.496 & 0.468 \\
\hline
\end{tabular}

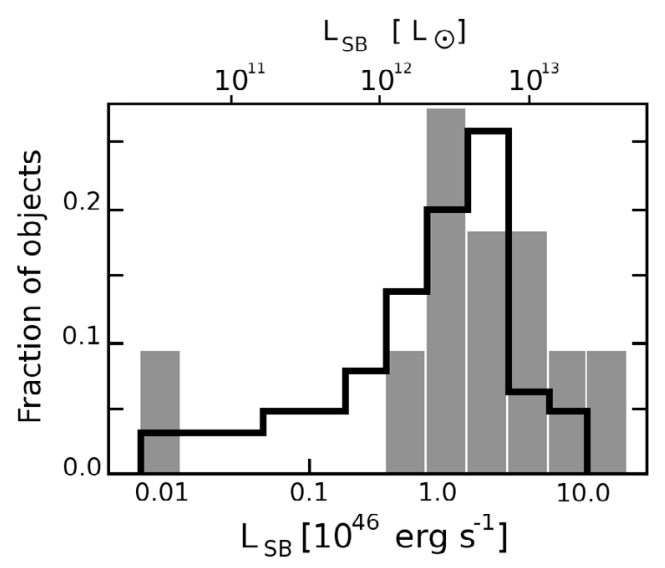

Fig. 4. Distribution of $L_{\mathrm{SB}}$ (integrated between 8 and $1000 \mu \mathrm{m}$ ), as an indicator of star formation, for type 1 (black) and type 2 (grey) AGN with $5 \sigma$ detections at $250 \mu \mathrm{m}$.

different types of AGN. Figure 4 shows the distribution of $L_{\mathrm{SB}}$, as an indicator of star formation activity, for type 1 (black) and type 2 (grey) AGN for objects with $5 \sigma$ detections at $250 \mu \mathrm{m}$. Because of the difference in number of AGN per type, the histogram shows the fraction of objects per type.

Type 2 AGN detected at $250 \mu \mathrm{m}$ tend to have on average a larger $L_{\mathrm{SB}}$, trend also observed in Fig. 3 but their numbers are too low to obtain a more robust estimate of this trend and to study the possible implications. The different criteria applied for the selection of type 1 and type 2 AGN (see Sect. 2), however, are rather unlikely to affect this result, as none of the criteria particularly favours objects with stronger star formation: the SDSS quasars and the X-ray selected type 2 AGN are biased, if anything, towards AGN-dominated objects; the Lacy at al. (2007) type 2 AGN sample consists of objects occupying the AGNdominated part of the $S_{8.0} / S_{4.5}$ vs. $S_{5.8} / S_{3.6}$ colour diagram (Hatziminaoglou et al. 2008); finally, the MIR selected type 1 and type 2 AGN have been chosen among $24 \mu \mathrm{m}$ emitters, but as was already shown in Fig. 1 the $24 \mu \mathrm{m}$ flux of AGN is dominated by the torus component.

\section{Conclusions}

Until recently, the study of type 1 and type 2 objects focused mainly on their X-ray-to-MIR emission, with the two extreme $\lambda$ ranges sampling the nuclear activity (X-rays) and hot dusty torus (MIR) emission, respectively, and the middle wavelengths (optical-to-near IR) defining whether an AGN would be classified as type 1 (unobscured) or type 2 (obscured). With Herschel-SPIRE we can now also accurately probe the cold dust component, heated by star formation, and look for differences between the types of active galaxies not only in the immediate environments of their nuclei but also in their hosts. The somewhat surprising lack of a strong correlation between the AGN intrinsic properties $\left(L_{\mathrm{acc}}\right)$ and the star formation activity as traced by $L_{\mathrm{SB}}$, at least up to $z \sim 2$, possibly an effect of the narrow span of our sample in $L_{\text {acc }}$, requires further investigation with larger AGN samples that also cover a wider accretion luminosity range, including stacking sources undetected by SPIRE. The same holds for the seemingly higher star formation activity in type 2 AGN. Even though this may not necessarily conflict with geometric unification schemes, it certainly opens the way for investigations in new directions.

Acknowledgements. SPIRE has been developed by a consortium of institutes led by Cardiff University (UK) and including Univ. Lethbridge (Canada); NAOC (China); CEA, LAM (France); IFSI, Univ. Padua (Italy); IAC (Spain); Stockholm Observatory (Sweden); Imperial College London, RAL, UCLMSSL, UKATC, Univ. Sussex (UK); and Caltech, JPL, NHSC, Univ. Colorado (USA). This development has been supported by national funding agencies: CSA (Canada); NAOC (China); CEA, CNES, CNRS (France); ASI (Italy); MCINN (Spain); Stockholm Observatory (Sweden); STFC (UK); and NASA (USA). The data presented in this paper will be released through the Herschel Database in Marseille $\mathrm{HeDaM}^{2}$. This work makes use of data taken with the Spitzer Space Telescope, the Sloan Digital Sky Survey (http://www.sdss.org) and the Two Micron All Sky Survey (http:// www. ipac. caltech. edu/2mass/overview/access.html). This work made use of Virtual Observatory tools and services for catalogue searches, crosscorrelation and plotting namely TOPCAT (http://www.star.bris.ac.uk/ mbt/topcat/), and VizieR (http://vizier.u-strasbg.fr/cgi-bin/ VizieR). E.H. would like to thank Kambiz Fathi and Jacopo Fritz for the very useful discussions. We thank the anonymous referee for the very insightful comments.

\section{References}

Abazajian, K. N., Adelman-McCarthy, J. K., Agüeros, M. A., et al. 2009, ApJS, 182,543

Beichman, C. A., Cutri, R., Jarrett, T., Stiening, R., \& Skrutskie, M. 2003, AJ, 125,2521

Bertelli, G., Bressan, A., Chiosi, C., Fagotto, F., \& Nasi, E. 1994, AAPS, 106, 275

Carilli, C. L., Bertoldi, F., Rupen, M. P., et al. 2001, ApJ, 555, 625 Condon, J. J., Cotton, W. D., Yin, Q. F., et al. 2003, AJ, 125, 2411 Elbaz, D., et al. 2010, A\&A, 518, L29

Fritz, J., Franceschini, A., \& Hatziminaoglou, E. 2006, MNRAS, 366, 767 Griffin, M. J., et al. 2010, A\&A, 518, L3

Hao, L., Weedman, D. W., Spoon, H. W. W., et al. 2007, ApJ, 655, L77 Hatziminaoglou, E., Fritz, J., Franceschini, A., et al. 2008, MNRAS, 386, 1252 Hatziminaoglou, E., Fritz, J., \& Jarrett, T. 2009, MNRAS, 399, 1206

Lacy, M., Storrie-Lombardi, L. J., Sajina, A., et al. 2004, ApJSS, 154, L166 Lacy, M., Petric, A. O., Sajina, A., et al. 2007, AJ, 133, 186 Levenson, N. A., Sirocky, M. M., Hao, L., et al. 2007, ApJ, 654, L45 Lonsdale, C., Polletta, M., Surace, J., et al. 2004, ApJS, 154, 54 Lutz, D., Sturm, E., Tacconi, L. J., et al. 2008, ApJ, 684, 853 Mainieri, V., Bergeron, J., Hasinger, G., et al. 2002, A\&A, 393, 425 Martínez-Sansigre, A., Rawlings, S., Lacy, M., et al. 2006, MNRAS, 370, 1479 Netzer, H., Lutz, D., Schweitzer, M., et al. 2007, ApJ, 666, 806 Oliver, S., et al. 2010, A\&A, 518, L21

Omont, A., Cox, P., Bertoldi, F., et al. 2001, A\&A, 374, 371 Papovich, C., Cool, R., Eisenstein, D., et al. 2006, AJ, 132, 231 Pilbratt, G. L., et al. 2010, A\&A, 518, L1

Poglitsch, A., et al. 2010, A\&A, 518, L2

Polletta, M., Wilkes, B. J., Siana, B., et al. 2006, ApJ, 642, 673

Priddey, R. S., Isaak, K. G., McMahon, R., \& Omont, A. 2003, MNRAS, 339, 1183

Richards, G. T., Lacy, M., Storrie-Lombardi, L. J., et al. 2006, ApJS, 166, 470

Riechers, D. A., Walter, F., Carilli, C. L., et al. 2006, ApJ, 650, 604

Schweitzer, M., Lutz, D., Sturm, E., et al. 2006, ApJ, 649, 79

Wang, R., Carilli, C. L., Wagg, J., et al. 2008, ApJ, 687, 848

2 http://hedam.oamp. fr /HerMES 
1 ESO, Karl-Schwarzschild-Str. 2, 85748 Garching bei München, Germany

e-mail: ehatzimi@eso.org

2 Institut d'Astrophysique de Paris, UMR 7095, CNRS, UPMC Univ. Paris 06, 98bis boulevard Arago, 75014 Paris, France

3 Centre for Astrophysics Research, University of Hertfordshire, College Lane, Hatfield, Hertfordshire AL10 9AB, UK

4 Dept. of Physics \& Astronomy, University of California, Irvine, CA 92697, USA

5 Institute for Astronomy, University of Edinburgh, Royal Observatory, Blackford Hill, Edinburgh EH9 3HJ, UK

${ }^{6}$ Cardiff School of Physics and Astronomy, Cardiff University, Queens Buildings, The Parade, Cardiff CF24 3AA, UK

7 Laboratoire AIM-Paris-Saclay, CEA/DSM/Irfu - CNRS Université Paris Diderot, CE-Saclay, pt courrier 131, 91191 Gif-sur-Yvette, France

8 Astrophysics Group, Imperial College London, Blackett Laboratory, Prince Consort Road, London SW7 2AZ, UK

9 California Institute of Technology, 1200 E. California Blvd., Pasadena, CA 91125, USA

10 Jet Propulsion Laboratory, 4800 Oak Grove Drive, Pasadena, CA 91109, USA

11 Laboratoire d'Astrophysique de Marseille, OAMP, Université Aixmarseille, CNRS, 38 Rue Frédéric Joliot-Curie, 13388 Marseille Cedex 13, France

12 Instituto de Astrofísica de Canarias (IAC) and Departamento de Astrofísica, Universidad de La Laguna (ULL), La Laguna, Tenerife, Spain

13 Dept. of Astrophysical and Planetary Sciences, CASA 389-UCB, University of Colorado, Boulder, CO 80309, USA
14 Herschel Science Centre, European Space Astronomy Centre, Villanueva de la Cañada, 28691 Madrid, Spain

15 Observational Cosmology Lab, Code 665, NASA Goddard Space Flight Center, Greenbelt, MD 20771, USA

16 Astronomy Centre, Dept. of Physics \& Astronomy, University of Sussex, Brighton BN1 9QH, UK

17 Dipartimento di Astronomia, Università di Padova, vicolo Osservatorio, 3, 35122 Padova, Italy

18 Institute of Astronomy, University of Cambridge, Madingley Road, Cambridge CB3 0HA, UK

19 Department of Physics \& Astronomy, University of British Columbia, 6224 Agricultural Road, Vancouver, BC V6T 1Z1, Canada

20 UK Astronomy Technology Centre, Royal Observatory, Blackford Hill, Edinburgh EH9 3HJ, UK

21 Institut d'Astrophysique Spatiale (IAS), bâtiment 121, Université Paris-Sud 11 and CNRS (UMR 8617), 91405 Orsay, France

22 Infrared Processing and Analysis Center, MS 100-22, California Institute of Technology, JPL, Pasadena, CA 91125, USA

23 School of Physics and Astronomy, The University of Manchester, Alan Turing Building, Oxford Road, Manchester M13 9PL, UK

24 Mullard Space Science Laboratory, University College London, Holmbury St. Mary, Dorking, Surrey RH5 6NT, UK

25 Space Science \& Technology Department, Rutherford Appleton Laboratory, Chilton, Didcot, Oxfordshire OX11 0QX, UK

${ }^{26}$ Institute for Space Imaging Science, University of Lethbridge, Lethbridge, Alberta, T1K 3M4, Canada

27 Astrophysics, Oxford University, Keble Road, Oxford OX1 3RH, UK 